Revue d'histoire de l'Amérique française

BSW REVUE D.HISTOIRE DE L'AMÉRIQUE FRANÇAISE

\title{
Inventaire des documents concernant l'histoire du Canada conservés aux Archives de Chicago (suite et fin)
}

\section{Honorius Provost}

Volume 4, numéro 4, mars 1951

URI : https://id.erudit.org/iderudit/801678ar

DOI : https://doi.org/10.7202/801678ar

Aller au sommaire du numéro

Éditeur(s)

Institut d'histoire de l'Amérique française

ISSN

0035-2357 (imprimé)

1492-1383 (numérique)

Découvrir la revue

Citer ce document

Provost, H. (1951). Inventaire des documents concernant l'histoire du Canada conservés aux Archives de Chicago (suite et fin). Revue d'histoire de l'Amérique française, 4(4), 591-600. https://doi.org/10.7202/801678ar d'utilisation que vous pouvez consulter en ligne. 


\section{BIBLIOGRAPHIE}

\section{INVENTAIRE DES DOCUMENTS CONCERNANT L'HISTOIRE DU CANADA CONSERVÉS AUX ARCHIVES DE CHICAGO}

(suite et fin)*

1699, oct. 2. Requête pour élection de tuteur aux enfants de Vaudreuil, alors gouverneur de Montréal. Signé Vaudreuil, Dupuy (x).

1699, oct. 13. D'Iberville 6crit de Larochelle à Nicolas Thoinard, à Paris, au sujet de son second voyage.

1700, janv. 28. Pierre de Cholenec, S.J. ré-échange de propriété à Montréal.

1700, janv. 29. Reçu et signature de René Brehán de la Galinière.

1700, fév. 3. Mgr de Laval donne procuration à Pierre Drouillard pour faire des concessions à l'Ile Jésus (x).

1700, juil. 13. L'abbé François Dollier, P.S.S. recommande Mme Mathurin Parent pour l'obtention d'une licence.

1700, août 21. Fragment d'acte notarié portant les signatures de Ramezay et Véron de Grandmesnil.

1701, mai 21. Lettre de la veuve Lesueur, Fort St-Louis des Ill. annonce à M. Blondeau, marchand de Montréal, la mort de son mari et de son frère St-Michel.

1701, sept. 1. Lettre de l'intendant Champigny à M. de Pontchartrain(x). 1701, nov. 1. François de Belmont, P.S.S. concède aux habitants de la côte St-Lambert le terrain d'un chemin pour venir à Montréal.

1702, mai 13. Contrat de société entre Charles Juchereau de St-Denis et François-Marie Bouat, à Montréal.

1702, nov. 29. Procès-verbal sur la guerre contre les Anglais, signé à LaMobile par Bienville, De la Salle, Boisbriant, De Tonty, etc.

1703, avril 16. Ordre au Sr de Maricourt pour chasser les Iroquois, signé Callière et scellé.

1703, avril 27. Acte de société entre les Srs Feyère et Desruisseaux, Montréal.

* Voir, Revue d'Histoire de l'Amérique française, IV, no 2 (septembre 1950): 294-302; IV, no 3 (décembre 1950): 453-458. 
1703, mai 6. Fragment de lettre avec signature de Pierre You de la Découverte.

1703, juil. 6. Reçu en parchemin signé à Paris par Mgr de St-Vallier. 1703, oct. 11. Contrat de mariage signé à St-François par le R.P. Jacques Bigot, Antoine de Planiol et Jean Legras.

1704, juin 1. Nomination de Juchereau Duchesnay au Conseil Supérieur de Québec; parchemin signé par Louis XIV (x, page lxxii).

1704, juin 3. Fragment d'un billet par A. Pacaud, Montréal.

1704, sept. 14. Permission par François de Beauharnois à Cochois de retourner au Fort Frontenac.

1705, juin 1. Billet promissoire de St-Germain à Simon Rhéaume.

1706, mars 8. Facture de marchandises, signée à Montréal par Lamothe Cadillac.

1706, nov. 3. Concession d'une seigneurie aux Frères Charons sur la rivière Yamaska, signée Callière et Champigny, et scellée.

1707, mai 4. Reconnaissance de dette par J.Bte Coutteau en faveur de Jacques Langlois, signée au Fort Pontchartrain; témoins Chesne et Alexis LeMoyne.

1707, juil. 27. Reçu de Hazeur en faveur de Delle de la Lory.

1707, août 9. Acte de société entre Pierre Chesne, Pierre Gareau et François Chesne; aussi signé par Jacques Lucas et A. Perrin.

1707, août 10. Lettre de Chesne, Fort Pontchartrain, à M. Nevesier, Montréal; affaires de commerce.

1707, oct. 17. Madeleine de Roybon d'Allonnes adresse une requête à l'intendant Raudot; réclamations au sujet du Fort Frontenac (x).

1707, oct. 19. Ordonnance de Raudot aux Messieurs de la Compagnie.

1708, mai 19. Acte de société entre Bertrand Arnaud et Joseph Desruisseaux.

1710, juil. 25. Reconnaissance de dette par Claude Parent et Jacques Langlois en faveur de François Trudeau, payable à Wabash.

1711, mai 21. Acte de société entre Jean Beauchamp et Daniel-Joseph Madoux.

1712, sept. 18. Réclamation contre Tonty par le Sr Parent (x).

1714, fév. 21. Reçu signé par le R.P. Frs Vaillant, S.J., à Montréal.

1714, août 3. Congé par Philippe de Rigaud de Vaudreuil à Québec, pour la traite aux Grands Lacs.

1715, mars 7. Engagement de Jacques Cardinal à Louis de Ramezay pour voyaige aux Outaouais.

1716, maì 3. Contrat de société entre Pierre Buteau et Pierre Biron, pour un voyage de traite à Michillimakinace.

1716, oct. 8. Reconnaissance des Pères Récollets comme missionnaires en l'Isle-Royale (Cap Breton); signée par De Costebelle.

1716, oct. 14. Dépêche au Ministre par Philippe de Rigaud de Vaudreuil, donnant rapport des événements dans son gouvernement en 1716 (x). 
1717, sept. Convention de Nicolaśs Rose, voyageur, pour conduire le Sr de Longueville à Kaskalskia.

1717. Engagement, à Ville-Marie, de Charles Nolan et Chs Lamarque au service de M. de Tonty, pour trois ans, au Fort Pontchartrain.

1718, août 28. Lettre ecrite, "Aux Illinois", par Jean-Bte et Louis Turpin à leur sœur.

1718, sept. 21. Acte de société signé à Montréal entre Paul Le Gardeur de St-Pierre et René Godfroi Sr de Linctot partant pour l'ouest.

1719, mai 5. Acte de société signé à Montréal entre Pierre Cóloron et Charles Sureau; nom du notaire enlevé.

1719, juin. Reconnaissance de dette par Delisle et Morin à FrançoisMarie Bouat; Testard de Montigny témoin.

1720, mars 12. Concession de terre à Montréal par le R.P. Pierre Lagrène, S.J.

$1720, . .$. "Relation d'un voyage fait de LaRochelle à la Louisiane par le Sr Fourcade, chirurgien du Roy."

1720-1721. "Relation d'un voyage que M. Le Page du Prat a fait de la Nouvelle-Orléans aux Natchez, sur le fleuve Mississipi."

1721, juin 6. Certificat par DuBuisson en faveur de Jacques HubertLacroix, à Montréal (x).

1722, mars 23. Procuration signée par Bienville, à Biloxi, en faveur du Sr Raguet dans la succession de Jacques de Ste-Hélène (x).

1722, juin 19. Instruction au Sr de Bourgmont par les directeurs de la Cie des Indes d'établir un poste sur le Missouri.

1722, juin. Procuration par le Sr Etienne de Villedonné, Montréal.

1723, avril 14. Échange et société entre Jacques Gamelin et Claude Caron, à la Baie des Puants, en présence de M. de Montigny, commandant, et du Père Chardon, S.J.

1723, juil. 16. Procuration de René d'Amours de Clignancourt, Michillimakinac, à Monier.

1723, juil. 20. Accord de traite, à Michillimakinac, entre Louis Prud'homme et les Srs Grandpré et Chambly.

1723, août 1. Cession par Françoise Juchereau de St-Denis, veuve de François de La Forest, à Alphonse Tonty, gouv. de Louisiane, concernant les frais de construction des forts Pimitoui, LaRoche et Chicagou; signée par Tonty et Charles Gervaise (x).

1723, août 1. Reconnaissance de dette par Henri Biron, en faveur de Jacques St-Denis, à Détroit.

1724, juil. 15. Accord entre Frs Amariton, Nicolas Sarazin et François Augé dit Lajeunesse, à la Baie, pour nommer comme procureur René de Couagne.

1724, juil. 22. Société pour la traite entre le Sr Deschaillons et Pierre Hubert dit Lacroix, à Michillimakinac.

1724, sept. 1. Testament de Jean Rivet, fait au Missouri.

1724, oct. 5. Contrat de Pierre Dugué de Boisbriant, à la Louisiane. 
1724, oct. 16. Inventaire des effets de l'intendant Edme-Nicolas Robert, mort en mer, en venant remplacer Bégon (36 pages de texte).

1724, ... Relation d'un voyage fait sur les rivières Mississipi, Missouri et Arkansas, à partir du Fort Orléans.

1725, janv. 15. Inventaire des effets de Jean Rivet, signé Louis de la Valtrie du Tisné, commandant à Kaskaskia, Hertel de Moncour et le Chevalier d'Ailleboust.

1725, mars 31. Reçu de Pierre Bourdon en faveur de François Bibeau et Antoine Robert; signé aussi par Claude Robillard.

1725, mai 30. Rȩ̣u du Sr Rhéaume en faveur de J.B. Desjardins, à Detroit.

1725, août 3. Contrat de mariage, à Michillimakinac, entre Jean Cauchois et Charlotte Chevalier, en présence de DeLignéry et du Père de St-Pé, S.J.; déposé ensuite dans le greffe du not. Dame (?).

1726, sept. 29. Lettre de recommandation au Comte Des Gouttes, pour le Ministre, signée à Québec par Beauharnois.

1726, ... Engagement de Joseph Brazeau à François You pour mener deux canots de marchandises par l'Outaouais à Michillimakinac; signe Despointes, St-Dizier, etc.

1727, mai 14. Contrat de société entre Louis Denys de la Ronde, commandant à la Pointe de Chay8amigon, Louis-Mathieu d'Amours et Nicolas d'Ailleboust.

1727, juin 6. Lettres patentes de la Compagnie des Sioux, passées à Montréal par Beauharnois, devant de Longueuil, de La Corne et d'Aigremont (x, page lxxii).

1727, sept. 3. Reconnaissance de dette en faveur du Sr Gamelin, de Montréal, par Duplessis Faber, commandant à la Baie des Puants.

1728, mai 29. Extrait d'une lettre à Beauharnois par le R.P. Guignas, S.J., de la mission du Fort de Beauharnois, pays de Sioux.

1729, fév. 22. Inventaire des papiers de feu le notaire Florent de la Cetière, à Québec; minute de Barbel (75 pages de texte).

1729, avril 6. Lettre de Picoté de Belestre, à Détroit, à son épouse, à Montréal; détails très importants sur personnes et choses.

1729, mai 24. Lettre de Cuillerier à sa mère, datée de Détroit.

1729, août 18. Réflexions sur la lettre de M. Périer, commandant de la Louisiane.

1729 (?). Association entre Zacharie Rabutel de La Noue, Paul de Lamarque et Marin pour traite avec les indiens.

1730, mars 22. Procuration par D'Arnaud pour M. de Francheville.

1730 , juin 1. Liste des effets du magasin de Québec livrés aux sauvages en diverses occasions durant 1729; signée Hocquart.

1730, oct. 19. Certificat de mérite pour St-Ours Deschaillons, après la prise de Haverhill, N.Angleterre, signé Beauharnois.

1730 (?). Engagement de Maurice Ménard à M. de Louvigny comme interprète dans une expédition. 
1731, avril 4. Engagement à Montréal de Louis Jetté et Ls Dufresne à Christophe Duf rost de la Jemmeraye pour les pays d'en haut.

1731, juin 4. Procuration de René Roy, Détroit, à Frs Filiatrault.

1731, juin 23. Engagement de Louis Marcheteau-Desnoyers à Louis Drouet, Sr de la Gravière, Montréal, pour faire un voyage en canot à Michillimakinac.

1731, août 15. Vente d'un esclave par Louis Chappeau à Pierre Guy (x). 1732, mai 9. Association entre Nicolas et François Volant, Montréal.

1732, juin 3. Association, à Détroit, entre D'Ainault, commandant au fort St-Philippe des Miamis et Pierre Roy, devant M. de Boishébert.

1732 , juin 4. Procuration signée Marin à son épouse.

1732, juin 9. Association entre Jacques-François Legardeur de Courtemanche et Didace Mouet pour traite à la Baie des Puants.

1732, oct. 2. Lettre de Charles Gaillard, seigneur de l'Ile d'Orléans, demandant à G. de Tonnancourt l'autorisation de vendre une propriété de Mme de Latouche.

1732, nov. 17. Certificat de capacité au Sr Jérôme, signé par St-Ange, commandant au Fort de Chartres.

1732, nov. 17. Contrat signé par Du Tisné.

1732, ... "Journal du Voyage que M. Régis de la Roullet a fait dans La Louisiane en 1732, de Mobile en allant aux Tchactas et des Tchactas à la Nouvelle-Orléans en descendant la Rivière aux Perles".

1732 (?). Obligation de Louise de Louvigny en faveur de Devaine.

1732 (?). Obligation de Michel Monette en faveur de Jacques Dugué.

1732-1733. Extraits du "Journal des Voyages faits par le Sieur Régis de la Roullet dans la Louisiane".

1733, juin 28. Reconnaissance par Jacques Cardinal, Détroit, en faveur de Laurent Parent et Pierre Hubert-Lacroix.

1734, mars 26. Procuration de Chs-Frs de Mézière en faveur de son épouse Suzanne Nolan, signée aux Ouatanons.

1734, mai 31. Lettre de Péan, à Détroit, à M. de Senneville, à Montréal, pour lui recommander François Barrois, qui va se faire soigner par M. Faure, curé de St-Sulpice.

1734, juin. Accord, à Montréal pour la traite, entre Pierre Hertel commandant à la Baie des Puants et Pierre Chartier.

1734, sept. 16. Information de l'envoi d'un chef indien aux autorités de la Martinique, signée Beauharnois et Hocquart (x).

1735, mai 13. Billet promissoire par Charles Marchand, à la Rivière St-Joseph, en faveur du Sr. Mogras.

1735, déc. 27. Ordre au Sr Hertel, de St-François, de rechercher les soldats déserteurs, signé Beauharnois (x).

1735-1741. Charles Fleury, chevalier: lettres établissant ses états de service (compagnon de d'Iberville en 1694) (x).

1736, juin 24. Lettre de Charles Fleury, explorateur canadien, au Ministre, de Larochelle. 
1737, juin 1. Ordre de Payen de Noyelle, Detroit, à Pierre Fortville de payer aux Srs J.B. Mallet, d'Aignau, Labutte et Navarre, et recus signés de ces derniers.

1737-1784. Documents du Fort de Chartres, de Kaskaskias, etc. concernant le commerce, les titres de propriété, etc. (deux liasses, en tout 135 pièces).

1738, mai 23. Engagement de Joseph Jérôme, de Laprairie, à ChsFrançois de La Pérade, commandant au poste des Miamis, pour conduire un canot de marchandises de Montréal à Détroit.

1738, mai 24. Procuration signée à Montréal, par Joseph de Tonty, commandant à la Baie des Puants, en faveur de son épouse.

1738, juin 4. Transport, à Montreal, du permis de traite de Louis Godefroy de Moranville, commandant aux Ou8atanons, à François Augé et Pierre Bissonnet, marchands de Montréal.

1738, août 6. Procuration par Claude-Ant. Bermen de la Martinière, commandant à la Baie des Puants, en faveur de Pierre l'Estage, marchand de Montréal.

1739, juil. 19. Engagement de Pierre Gaboury au Sr Marin; minute d'Adhémar, not. à Montréal (coté $\$ 250.00$ ).

1739-1740. Journal de la Guerre du Mississipi contre les Chicachas en 1739 et terminée en 1740. Manuscrit des papiers de Philippe Buache, géographe du Roi.

1739-1746. Projet de Philippe Buache pour la publication de cartes, en 1739, avec une lettre à d'Argenson, Ministre, et la réponse de ce dernier, en 1746.

1742, déc. 9. Reçu du R.P. Luc-Frs Nau, S.J. en faveur de François Desroussons, Sault-St-Louis.

1742, déc. 20. Consentement pour échange de propriêté, signé à Montréal par le Père D. Richer, S.J.

1743, mai 31. Résiliation de société entre le chevalier de Bertel, commandant aux Illinois, et De La Loève, Flaucourt et Desruisseaux.

1743, ... Lettre de Louis Lefebvre Du Chouquet au Lieut. Gén. à Montréal, affaires de traite.

1744, juil. 14. Procuration par J -Claude Marin, Sr de Laperrière, à St-Joseph, en faveur de Pierre Hervieux.

1744, nov. 15. Extrait des registres du Gouv. de Québec, concernant la propriété des Mles Mingan par les héritiors Jolliet; signé Beauharnois et Hocquart.

1745, juin 9. Contrat entre le Sr Laduc et Joseph Petit, à Montreal.

1745, août 6. Lettre de J.Bte Parent, Détroit, à son frère.

1746, août 18. Procuration de J.-Bte Guyon à Pierre LeDuc; témoin, De Noyelle, commandant à Michillimakinac.

1746, sept. 29. Lettre de Beauharnois et Hocquart au Ministre; traite de la conduite de la guerre (20 pages). 
1747, janv. 16. Vente par François Dugaux à Pierre Marcheteau, devant le not. Pillard, Tr. Rivières.

1747, fév. 12. Copie contemporaine de la capitulation accordée aux Anglais par Coulon de Villiers, à Grand-Pré.

1747, mars 9. Quittance par Villiers de Jumonville au Sr Soumande Delorme, minute d'un notaire de Montreal.

1747, avril 4. Contrat de Louis d'Amours, Sr de Clignancourt, avec le Sr Lemoyne de Monière (x).

1747, juin 1. Instructions données par La Vérandrye au Sr Gamelin pour réparer les Forts Maurepas et La Reine; fait à Montréal (x).

1747, juin 3. Reçu signé de Robineau de Bécancourt (annexé à une vente du 16 janv. 1747).

1747, août 16. Procuration du Sr. François dit Larose en faveur du Sr Baribeau, à Michillimakinac.

1747, ... Contrat de vente signé par La Galissonnière, gouv.

1748, janv. 25. Dotation de la Sœur Marin; signatures de Marin, La Galissonnière, etc. Minute d'Adhémar, not. à Montréal.

1748, mars 29. Vente de Chs Marchand à son nevea Foucher, à StJoseph de Lévis.

1749, mai 26. Reçu d'Etienn, Dumont, commandant aux Miamis, en faveur de M. Levine, Montréal.

1749, juin 1. Procuration donnée, à Montréal, à Ignace Gamelin, marchand, par M. de Sabrevois, la veille de son départ pour le Fort Pontchartrain.

1749, juin 19. Billet promissoire de Frs Jolliet à Ignace Gamelin.

1749, juil. 3. Billet de concession par St-Ange à Baptiste Racine, au poste de Vincennes.

1749, oct. 23. Ordre de La Jonquière à Hertel de partir pour Louisbourg.

1749 (?). Lettre écrite à Détroit par Louise Tessier, épouse de Pierre Payet, à son frère Tessier dit Choumine.

1750, juin 13. Contrat entre François Dubreuil, Sr de La Corne, et Louis Coulons de Villiers, à Montréal.

1750 (circa). Lettre de Joseph Jolliet à sa mère.

1750-1751. Volumineux dossier judiciaire dans l'affaire de Antoine Juchereau-Duchesnay contre Lanouillier des Granges.

1751, juin 6. Résiliation de société entre Pierre de Tailly et le Sr Marsolet, au fort St-Joseph.

1751, juil. 19. Réponse de Paul Lanouillier, juge prévôt de Beauport, contre Antoine Juchereau-Duchesnay; aussi signée: Saillant et Thibault.

1752, août 3. Paul-Joseph de Longueuil écrit de Détroit: "Je pars aujourd'hui pour la Baie des Puants..." Questions d'affaires.

1752, sept. 28. Inventaire, à Michillimakinac, des effets de feu le $\mathrm{Sr}$ Marin de Laperrière, en présence du R.P. DuJaunoy, de M. de Beaujeu, commandant, de Langlade, Bourassa et Baribeau, résidents. 
1752 (?). Instructions de M. de Longueuil à M. Vassan, suivant celles de Jonquière (x).

1753, oct. Louis Hertel nommé cadet dans l'armée, à Québec, signé Duquesne (x).

1754, avril 27. Lettre de louange au sauvage En8esté, signée Duquesne.

1754, juil. 4. Lettre de louange au sauvage Sapanossa, signée Duquesne.

1754, août 30. "Précis de la suite des considérations sur les nouvelles découvertes au Nord de la Grande Mer”, présenté au Roi par Philippe Buache, géographe.

1754, déc. 7. Décret de Louis XV sur le gouvernement de Métis.

1755, mars 6. Diplôme de notaire public donné à Thantaloup par d'Auberville, Nouvelle-Orléans.

1755, mars 13. Reçu signé par Alex. Berthier, interprète, à la NouvelleOrléans.

1755, mai 9. Reçu de paiement signé James Wolfe (x).

1755, mai 15. Reçu signé par François Sarazin, interprète, à la NouvelleOrléans.

1755, juin 15. Lettre de Shirley à Bradstreet, alors à Oswego.

1755, juil. 9. Liste des officiers tués ou blessés sur les bords de la Monongahéla.

1755, oct. 20. Conseil de guerre par le Gén. Johnson, au lac George.

1755, ... Discours du Gouv. Wentworth Benning à l'ouverture de la Législature du N. Hampshire, où il est question de la guerre du Canada et de l'expédition de Crown Point.

1756, sept. 20. Lettre de Pierre Monbron et de son épouse, à Michillimakinac, à leur fille.

1757, avril 10. Transport des droits de traite par St-Ange de Beaucour et Michel de Villebois à Boisberthelot de Beaucour, gouv. de Montréal.

1757, mai 20. Engagement, à Montréal, d'Amable Jérôme, de Laprairie, à Jordy de Villebois, pour le Grand Portage.

1757, juin 27. Procuration à Joseph Fleury Deschambault par Pierre Leduc et Ls Hurtubise, à Montréal, devant Jean le Verrier.

1757, août 18. Lettre de Montcalm, signée au Portage (x).

1757, sept. 30. Abandon, à Montréal, par Pierre Rigaud de Vaudreuil de ses intérêts de traite à la Baie des Puants, en faveur de Jacques Giasson et Ignace Hubert (x).

1758, mai 24. Acceptation par Le Gardeur de Repentigny du Sr Deschambault des postes de Kaministig8ia et Michipicotin avec les effets qu'ils contiennent.

1758, août 8. Lettre du Chev. de Lévis à Melfort (x).

1758-1759. "Cahiers d'extraits des registres civils de la jurisdiction des Trois-Rivières" (50 pages); Signés Pillard, Hertel de Rouville, etc. 1759, janv. 1. Nomination du Sr Hertel, de St-François, enseigne en pied au Canada; signée pár Louis XV. 
1759, janv. 14. Vente par Joseph Dufour dit Latour à Pierre Rigaud de Vaudreuil, d'un terrain à Montréal (x, daté 31 juil. 1759).

1760, janv. 16. Nomination de Jacques Allier comme juge pour les paroisses de Berthier à Kamouraska incl.; signée par Murray et Cramahé, et scellée $(x)$.

1761, fêv. 16. Lettre de Roubaud à Murray(?) sur les dispositions des Abénaquis de St-François.

1761-1798. Registres paroissiaux du Fort de Chartres et de la Prairie du Chien.

1762, mars 2. Procuration donnée par Dame Josephte Soumande, à la Nouvelle-Orléans, à Joseph Fleury Deschambault, de Montréal, pour percevoir une dette du Sr St-Ange; témoins Hertel de Rouville, son époux, Ls-Nicolas Leduc, Marie LeNormand.

1763, janv. 25. Billet de concession signé par St-Ange, au poste de Vincennes.

1763, juil. 16. Lettres patentes de Louis XV créant le Sieur de Niverville chevalier de l'Ordre de Saint Louis. Albany.

1763, oct. 12. Lettre de Lacorne de St-Luc, Montréal, à Jansson, à

1763, déc. 2. Boucherville; congé par le L. Col. Baron de Munster à Thomas Dunhart, caporal au 4èmë bataillon, 60ème Royal Americans, dont Jeffrey Amherst est commandant en chef.

1764, avril 10. Buache, Philippe de, géographe du Roi. "Mémoire remis à l'Académie Royale de St-Pétersbourg..."

1764, sept. 20. Autorisation de James Murray à George Ross, à Londres, de payer le prix d'une montre en or à M. Dingwall (x).

1766, mars 28. Sir William Johnson à George Croghan.

1767, ... Shelbourne, de Whitehall, à Sir William Johnson.

1768, juin 1. Guy Carleton. Permission à l'abbé Pierre Gibault et instruction aux officiels de faciliter son voyage dans l'ouest. Datée de Montréal (x).

1769, déc. 9. Guy Carleton. Ordre à M. Allsoppe de livrer des provisions à un parti d'Iroquois.

1770, juil. 31. Guy Carleton. Ordre aux officiers d'ordonnance de livrer des marchandises aux indiens de Lorette $(x)$.

1770, déc. 18. James Stevenson, commissaire à Détroit, à sir William Johnson, concernant les François et indiens de l'endroit.

$1770, \ldots$ Col. John Wilkins; lettre autographe sur la guerre francoindienne.

1778, sept. 17. Autorisation de Frédéric Haldimand à John Powell, pour paiement à John Campbell, surintendant des affaires indiennes.

1782, sept. 8. Testament de l'abbé Pierre Gibault, fait à Ste-Geneviève.

1793-1833. Papiers concernant le Haut-Canada (70 documents), la fondation de Toronto, la guerre de 1812, etc. Signatures des Gouv. Simcoe, Russell, Hunter, etc. 
1795, oct. 25. Edouard, duc d'York. Liste de paye des travailleurs du $7 e ̀ m e$ régiment des Fusilliers Royaux, à Halifax.

1795, nov. 22. Edouard, duc d'York. Autorisation pour paiement adressée à Henry Atkins, payemaitre des dépenses contingentes, à Halifax.

1797, ... Lettre autographe de Joseph Brandt (Thayandanega), chef mohawk; affaires personnelles.

1798-1799. Comte Joseph de la Puisaye. Lettre au gouvernement et liste d'effets reçus pour les émigrants français de Windham, Ont.

1800, fév. 15. Lieut. gén. P. Hunter, Québec, à John McGill, concernant les émig rants français.

1800, mars 24. Lettre à James Green: affaires du jour à Québec.

1804, oct. 15. Le Commissaire des Comptes publics demandant compte à l'Hon. Peter Russell des sommes tirées sur le Trésor pour des provisions. 1804, ... Description de concessions de terres dans le H. Canada.

1807, mars 21. W. McGillivray, Montréal, à M. Cowan, questions de commerce.

1807, avril 21. Lettre de James Green à John McGill.

1807, mai 19. Lettre du même au même.

1807, oct. 3. L'Hon Francis Gore à John McGill à propos de fournitures.

1807, nov. 20. W. Thornton, Secrétaire militaire, au même, même sujet.

1809, fév. 5. Le même au même, même sujet.

1811-1813. Tableau des provisions, armements et habillements des troupes et des Indiens, dans le Haut-Canada.

1812, ... Papiers Dearborn (Maj. Gén. Henry): 113 documents militaires de la guerre de 1812; correspondances avec les généraux Ván Ranselaer, Wadsworth, Lewis, Bloomfield, etc.

1814, mars 14. Gordon Drummond, administrateur du Haut Canada. Ordre signé à York (Toronto), à John McGill, de payer à George Lowe le montant des dépenses contingentes du Conseil Législatif.

1814, déc. 26. Gordon Drummond, Administrateur, à Kingston. Ordre à John McGill de payer à Catherine Wolfrum une année de pension.

1818, janv. 15. Alexander Henry, explorateur, Montréal, à John Henry, affaires personnelles.

1823, oct. 10. Concession de terre à Alexander Wilkinson, dans la ville de Cornwall.

1827, juin 7. James Cowan, Fort Wayne, à son oncle Isaac Cowan, à Vaudreuil, affaires de famille.

1833, nov. 27. Concession de terre à Christy Grant, dans le comté de Glengarry.

Honorius Provost, ptre 